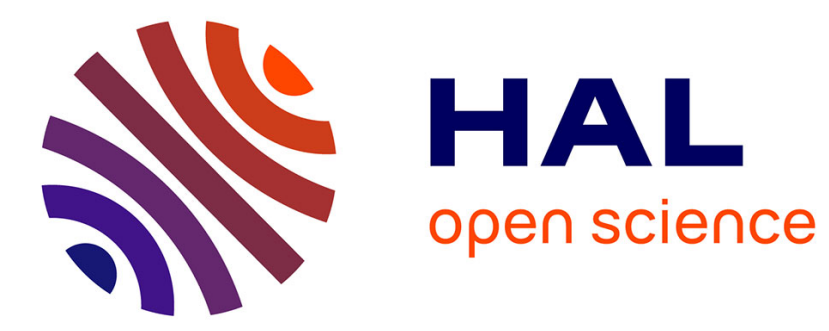

\title{
Répertoire des textes législatifs et réglementaires publiés pendant les mois de mai à octobre 1987 - Jurisprudence
}

\author{
Francis Meyer, Gilles Mathis
}

\section{To cite this version:}

Francis Meyer, Gilles Mathis. Répertoire des textes législatifs et réglementaires publiés pendant les mois de mai à octobre 1987 - Jurisprudence. 1988, pp.65-68. 10.4267/2042/25868 . hal-03424594

\author{
HAL Id: hal-03424594 \\ https://hal.science/hal-03424594
}

Submitted on 10 Nov 2021

HAL is a multi-disciplinary open access archive for the deposit and dissemination of scientific research documents, whether they are published or not. The documents may come from teaching and research institutions in France or abroad, or from public or private research centers.
L'archive ouverte pluridisciplinaire HAL, est destinée au dépôt et à la diffusion de documents scientifiques de niveau recherche, publiés ou non, émanant des établissements d'enseignement et de recherche français ou étrangers, des laboratoires publics ou privés. 


\section{législation et \\ jurisprudence}

\section{RÉPERTOIRE DES TEXTES LÉGISLATIFS ET RÉGLEMENTAIRES PUBLIÉS PENDANT LES MOIS DE MAI À OCTOBRE 1987}

\section{CHASSE}

\section{- Décrets}

Décret $n^{\circ} 87-440$ du 19 juin 1987 modifiant le décret $n^{\circ}$ 86-330 du 7 mars 1986 portant institution de conseils départementaux de la chasse et de la faune sauvage.

(J.O. du 25 juin 1987, p. 6857).

Décret $n^{\circ}$ 87-450 du 29 juin 1987 modifiant le décret $n^{\circ} 75-293$ du 21 avril 1975 fixant les règles d'exploitation de la chasse sur le domaine public maritime et sur la partie des cours d'eau domaniaux situés à l'aval de la limite de salure des eaux.

(J.O. du 30 juin 1987, p. 7021 ).

Décret du 6 octobre 1987 portant nomination de M. Pouget comme président du Conseil d'Administration de l'Office national de la Chasse.

(J.O. du 7 octobre 1987, p. 11689).

\section{- Arrêtés}

Arrêtè du 13 mai 1987 modifiant l'arrêté du 18 septembre 1975 portant statut des fédérations departementales des chasseurs.

(J.O. du 2 juin 1987, p. 5968).
Arrêté du 11 juin 1987 fixant la liste des oiseaux protéges sur tout le territoire national, y compris les terres australes et antarctiques françaises.

(J.O. du 9 juillet 1987, p. 7480).

Arrêté du 26 juin 1987 fixant la liste des espèces de gibier dont la chasse est autorisée. (J.O. du 20 septembre 1987, p. 10942).

Arrêtè du 26 juin 1987 instituant un plan de chasse pour certaines especes d'oiseaux d'eau.

(J.O. du 20 septembre 1987, p. 10942).

\section{DÉFRICHEMENT}

\section{- Décret}

Décret $n^{\circ} 87-715$ du 27 août i987 et circulaire du 27 août 1987 relatifs à la taxe sur le defrichement.

(Modification de certains articles du Code forestier).

(J.O. du 30 août 1987, p. 9983). 


\section{D.F.C.I.}

- Lois

Loi $n^{\circ} 87-565$ du 22 juillet 1987 relative à l'organisation de la sécurité civile, à la protection de la forêt contre l'incendie et à la prévention des risques majeurs.

(J.O. du 23 juillet 1987, p. 8199 et rectificatif J.O. du 29 août 1987, p. 9912).

\section{ÉCONOMIE FORESTIĖRE}

\section{- Arrêtė}

Arrêté du 20 août 1987 portant création du baccalauréat professionnel section Productique Bois et fixant les modalites de préparation et de delivrance de ce baccalauréat.

(J.O. du 5 septembre 1987, p. 10280).

\section{F.F.N.}

\section{- Arrêté}

Arrêté du 26 juin 1987 relatif aux subventions du Fonds forestier national pour les entreprises de travaux syivicoles, d'exploitation forestière et de scieries.

(J.O. du 9 août 1987, p. 9064).

\section{FORÊT MÉDITERRANÉENNE}

- Arrêté

Arrêté du 28 octobre 1987 relatif au Conseil d'orientation de la forêt méditerranéenne.

(J.O. du 30 octobre 1987, p. 12624).

\section{O.N.F.}

\section{- Décret}

Décret $n^{\circ}$ 87-679 du 17 août 1987 fixant la liste des forêts et terrains à boiser ou à restaurer appartenant à l'État, dont la gestion et l'équipement sont confiés à l'Office national des Forêts.

(J.O, du 19 août 1987, p. 9522).

\section{PẼCHE FLUVIALE}

\section{- Décrets}

Décret $n^{\circ}$ 87-388 du 10 juin 1987 pris pour l'application de l'article 437-10 du Code rural et déterminant les conditions de classement des cours d'eau, canaux et plans d'eau en deux catégories.

(J.O. du 16 juin 1987, p. 6417).

Décret $n^{\circ}$ 87-505 du 3 juillet 1987 modifiant le décret $n^{\circ}$ 85-1369 du 20 décembre 1985 pris en application de l'article 435 du Code rural et fixant les conditions dans lesquelles la pêche est interdite en vue de la protection du poisson.

(J.O. du 9 juillet 1987, p. 7480).

Décret $n^{\circ} 87-719$ du 28 août 1987 pris pour l'application de l'article 419 du Code rural et fixant les conditions d'exploitation du droit de pêche de l'État.

(J.O. du 2 septembre 1987, p. 10091).

\section{- Arrētés}

Arrêtè du 28 août 1987 portant modification du Code du domaine de l'Etat et relatif à l'exploitation du droit de pêche de l'État dans les eaux mentionnées à l'article 419 du Code rural.

(J.O. du 2 septembre 1987, p. 10088).

Arrêté du 16 octobre 1987 portant nomination au Conseil supérieur de la pêche. (Représentants de l'Agriculture: MM. Olivier Jean-Yves et Augier Pascal en remplacement de MM. Dutruc-Rosset Georges et Guillaume Benoit).

(J.O. du 24 octobre 1987, p. 12430). 


\section{PERSONNELS}

\section{- Arrêté}

Arrêté du 5 mai 1987 modifiant l'arrêté du 2 juillet 1985 instituant les commissions administratives paritaires compètentes à l'égard des corps des Ingénieurs des Travaux des Eaux et Forêts.

(J.O. du 22 mai 1987, p. 5603).

\section{POURSUITES}

\section{- Arrêté}

Arrêté du 5 juin 1987 relatif au traitement automatisé des contraventions relevant de la procedure de l'amende forfaitaire. (Concerne les contraventions des 4 premières classes... à la réglementation sur les parcs nationaux, à la réglementation intéressant les bois, forêts et terrains à boiser).

(J.O. du 25 juin 1987, p. 6856).

\section{PROTECTION DE LA NATURE}

\section{- Circulaire}

Circulaire du 11 mars 1987 relative à l'inspection des installations classées pour la protection de l'environnement.

(J.O. du 2 mai 1987, p. 4881).

F. MEYER

\section{JURISPRUDENCE}

\section{- CONTENTIEUX PÉNAL}

Affaire S.... Construction à distance prohibée - Infraction à l'article L. 151-2 du Code forestier Caractère successif de l'intraction. Arrêt de la cour d'appel de Colmar du 26 tévrier 1987.

"Sur les constructions sans autorisation en forêt domaniale soumise au régime forestier et à distance prohibée de la forêt (articles L. 151-2 et R. 151-2 du Code forestier):

Attendu que, contrairement au régime des poursuites pour défaut de permis de construire, prévu par le Code de l'urbanisme, les articles L. 151-1 et suivants ont créé des délits successifs dont le délai de prescription ne court qu'à partir de l'enlèvement de la construction irrégulière (cass. crim. 22 mai 1840 D-jur. gén. V Vo-Forêt $n^{\circ} 932$; cass. crim. 9 septembre 1847, Dalloz périodique $47,4,407) \cdots$.

Condamnation, pour la construction d'une baraque en forêt domaniale et d'une autre à distance prohibée, à deux amendes de $1200 \mathrm{~F}$, et ordre de démolir ou enlever les abris.

\section{Observations :}

Les dispositions du Code forestier régissant les constructions à distance prohibée, qui n'ont quasiment pas changé depuis l'origine du Code, ne donnent pas lieu à une jurisprudence abondante.

II est donc intéressant de relever cet arrêt de la cour d'appel de Colmar, qui confirme le caractère "successif " - ou " permanent " - de l'infraction en application d'une jurisprudence ancienne de la Cour de cassation et alors que les premiers juges y avaient vu une infraction instantanée. Le caractère successif de l'infraction, qui signifie qu'elle est réputée commise tant 
que l'effet délictueux ne cesse pas, permet d'en poursuivre la répression jusqu'à l'expiration du délai de prescription de l'action publique qui n'est décomptè qu'à partir, en l'occurrence, de l'enlèvement de la construction.

Il faut noter qu'à la différence de cette infraction forestière, le délit de construction sans permis, prévu et réprimé par le Code de l'urbanisme (article L 480-4), ne constitue pas une infraction successive.

\section{- CONTENTIEUX ADMINISTRATIF}

Affaire de L.... Défrichement - Demande d'autorisation - Retrait de la demande. Conseil d'État 27 mars 1987.

"Considérant que, par lettre recommandée en date du ......, adressée au directeur départemental de l'Agriculture des Alpes-Maritimes, M. de L.... a retiré sa demande ...... d'autorisation de défricher un terrain lui appartenant ...... ; que l'administration a néanmoins poursuivi l'instruction de la demande et que le ministre a rejeté cette demande par une décision du ....... ;

Considérant que, $M$. de L.... ayant purement et simplement retiré sa demande, le ministre ne pouvait que prend:e acte de ce retrait et ne pouvait légalement statuer sur la demande; que M. de L.... est dès lors fondè à demander l'annulation de la décision ..... ; " (annulation).

\section{Observations :}

II peut sembler évident que l'autoritẻ administrative soit dessaisie d'une demande d'autorisation que son auteur retire. Telle n'était pourtant pas, en matière de défrichement, la doctrine de l'administration, qui estimait qu'une fois le dossier enregistré, la demande était intangible et irréversible.

Cette conception, dérogatoire au droit commun, était principalement fondée sur deux considèrations. La première est que la procèdure de l'autorisation de défrichement peut conduire à une autorisation tacite: dans certaines circonstances où le retrait prêterait à contestation, l'autorisation tacite se trouverait acquise contre l'intention de l'administration. La seconde raison réside dans le caractère réel, et non personnel, de la décision, qui suit le fonds et est opposable aux propriétaires successifs, jusqu'à ce qu'un changement dans les éléments de fait ou de droit justifie qu'une décision différente y soit substituée.

L'administration des forêts estimait en outre faire application d'une règle, exprimée par circulaire au siècle dernier, selon laquelle "la déclaration visee à la sous-préfecture étant la seule base officielle du rapport (ou procès-verbal de reconnaissance), les agents forestiers ne doivent accueillir aucune modification verbale ou écrite de son contenu."

Le conseil d'État n'a pas retenu ces arguments, mais a entendu faire application du principe général. Une demande d'autorisation de défrichement est donc réversible.

On ne saurait, pour autant, en déduire qu'elle peut être, à tout moment de la procédure, modifiée, car la modification est d'une nature diffèrente du retrait; la régle à laquelle se référait l'administration en l'espèce, si elle était sans effet sur un cas de retrait de la demande, peut trouver à s'appliquer en cas de modification.

\section{G. MATHIS}

Chef du Bureau des Affaires juridiques DIRECTION DE L'ESPACE RURAL ET DE LA FORÊT

1ter, avenue de Lowendal

75007 PARIS 